\title{
RELAÇÕES ENTRE A EXPOSIÇÃO AOS AGRATÓXICOS E O DESENVOLVIMENTO DO CÂNCER: REVISÃO SISTEMÁTICA
}

Júlia Brangioni Fontes, Lindisley Ferreira Gomides, Pollyanna Álvaro Ferreira Spósito. Relações entre a exposição a agrotóxicos e o desenvolvimento do câncer: revisão sistemática. Revista Saúde Dinâmica, vol. 9, núm. 3, 2021. Faculdade Dinâmica do Vale do Piranga.

\section{SAÚDE DINÂMICA - Revista Científica Eletrônica FACULDADE DINÂMICA DO VALE DO PIRANGA}

9a Edição 2021 | Ano III - no 3 | ISSN - 2675-133X 


\title{
Relaçães entre a exposição aos agrotóxicos e o desenvolvimento do câncer: revisão sistemática
}

\author{
Relationships between pesticides exposure and development of cancer: systematic review \\ Júlia Brangioni Fontes ${ }^{1}$, Lindisley Ferreira Gomides ${ }^{2,3}$, Pollyanna Álvaro Ferreira Spósito ${ }^{2,3}$ \\ ${ }^{1}$ Discente do Mestrado em Ensino de Ciências da Saúde - Faculdade Dinâmica do Vale do Piranga \\ ${ }^{2}$ Docente Permanente do Programa de Mestrado em Ensino de Ciências da Saúde e do Ambiente (PROCISA) da \\ Faculdade Dinâmica do Vale do Piranga (FADIP) \\ ${ }^{3}$ Docente das Escolas de Medicina e Farmácia da Faculdade Dinâmica do Vale do Piranga (FADIP) \\ Autor correspondente: juliabrangioni@gmail.com
}

\section{Resumo}

A associação causal entre exposição ocupacional aos agrotóxicos e carcinogênese se estende para diversos agentes químicos e circunstâncias de trabalho, o que sugere uma correlação entre a expansão do uso dos agrotóxicos no país com o crescente aumento de casos de câncer, principalmente em agricultores. O objetivo do estudo foi verificar a relação entre exposição aos agrotóxicos e desenvolvimento de neoplasias e os impactos gerais na saúde do homem. Trata-se de uma revisão sistemática realizada no PubMed e no SciELO, utilizando os termos "Cancer AND Agrochemicals" e "Cancer AND Agrochemicals AND Farmworkers", em inglês e português. $\mathrm{O}$ estresse oxidativo agudo ou de longa duração perturba o metabolismo celular e pode acelerar o desenvolvimento de várias doenças, como o câncer. Observou-se riscos aumentados para neoplasias de mama, próstata, tireoide, lábio, pâncreas, linfomas e melanomas entre trabalhadores rurais e populações potencialmente expostas a agrotóxicos, embora sejam necessários mais estudos na área. Alguns vieses foram observados como a classificação incorreta de exposição não diferencial e o fato de a neoplasia ser uma doença multifatorial. Espera-se que essas discussões sejam incentivo para pesquisas na área, proporcionando visibilidade às questões relacionadas aos riscos eminentes da exposição aos agrotóxicos.

Palavras-chave: Agrotóxicos; Câncer; Neoplasia; Saúde do trabalhador rural.

\begin{abstract}
The causal association between occupational exposure to agrochemicals and carcinogenesis is related to various chemical agents and work circumstances, which suggests a correlation between the expansion of agrochemicals use in the country with the growing increase in cancer cases, mainly in farmworkers. The study aimed to verify the relationship between exposure to agrochemicals and development of neoplasms, and general impacts on human health. This is a systematic review carried out in PubMed and SciELO, using the terms "Cancer AND Agrochemicals", and "Cancer AND Agrochemicals AND Farmworkers", in English and Portuguese. The acute or long term oxidative stress disrupts cell metabolism and can accelerate the development of various diseases such as cancer. Increased risks for breast neoplasm, prostate, thyroid, lip, pancreas, lymphomas and melanomas were observed among rural workers and populations potentially exposed to pesticides, although are needed more studies in the area. Some biases were observed, such as the incorrect classification of non-differential exposure and, above all, the fact that neoplasm is a multifactorial disease. It is expected that the discussions will encourage other researches in the area, providing visibility to issues related to the eminent risks of exposure to agrochemicals.
\end{abstract}

Key words: Agrochemicals; Cancer; farmworker health. 


\section{INTRODUÇÃO}

A agricultura é uma prática existente na sociedade há mais de 10 mil anos, entretanto, quando se fala em uso de agrotóxicos para controle de pragas e de doenças em lavouras, essa prática existe há pouco mais de meio século, quando após as grandes guerras mundiais as indústrias fabricantes de venenos utilizados como armas químicas encontraram na agricultura um novo mercado para os seus produtos (LONDRES, 2015).

O uso de agrotóxicos no Brasil é regido pela Lei $N^{\circ}$ 7.802, de 11 de julho de 1989 (Lei dos Agrotóxicos), regulamentada pelo Decreto n ${ }^{\circ}$ 4.074/2002. A definição do termo é ampla, entretanto, de acordo com o seu Artigo $2^{\circ}$, consideram-se: "I - agrotóxicos e afins: a) os produtos e os agentes de processos físicos, químicos ou biológicos, destinados ao uso nos setores de produção, armazenamento e beneficiamento de produtos agrícolas cuja finalidade seja alterar a composição da flora ou da fauna, a fim de preservá-las da ação danosa de seres vivos considerados nocivos (grifo próprio).” A partir desse conceito, muitas divergências podem ter ocorrido pela falta do conhecimento do termo oficial "agrotóxico e afins", que já foram denominados como "defensivo agrícola", "praguicidas" ou "pesticidas", e até como "produto fitossanitário". O uso desses termos demonstra a fragilidade do conceito que, ao "embutir" a toxicidade do produto, permite conotações muitas vezes opostas ao seu sentido real, de acordo com os interesses dos envolvidos (LOPES, 2018).

A denominada "Revolução Verde", ocorrida na década de 1950, teve como seu principal trunfo o aumento do uso de agrotóxicos e permitiu a transformação da produção agrícola no mundo (RIBEIRO et al., 2018). No Brasil, essa revolução ocorreu em meados de 1970, com a implantação do Programa Nacional de Defensivos Agrícolas (PNDA). Com a promessa de crescimento da produção, associada a uma série de incentivos e isenções fiscais para a fabricação e comercialização de agrotóxicos, os "defensivos agrícolas" se tornaram um grande atrativo para a produção (OLIVEIRA et al., 2017) e o país rapidamente passou a figurar como um dos maiores consumidores do mundo em uma curva de crescimento exponencial (RIGOTTO, 2016). A Agência Nacional de Vigilância Sanitária (ANVISA, 2012) indicou que, nos últimos dez anos, o mercado mundial desses produtos cresceu 93\%, enquanto a taxa de crescimento no Brasil foi de 190\%, principalmente nos últimos cinco anos, nos quais o número de "defensivos agrícolas" aprovados cresceu significativamente. 
Com o crescimento do uso, consumo e fabricação de agrotóxicos, cresceu também o número de casos de doenças associadas a exposição a esses produtos (LOPES; ALBURQUERQUE, 2018). Estudos toxicológicos, clínicos e epidemiológicos apontaram associação entre a exposição aos agrotóxicos e diversas doenças, inclusive as neoplasias e as que envolvem base hormonal (PLUTH, 2017; SANTOS et al., 2018). Atualmente, a associação causal entre exposição ocupacional e carcinogênese se estende para mais de 40 agentes químicos, misturas e circunstâncias de trabalho, como grande exposição aos agentes químicos, negligência em relação ao uso de equipamentos de proteção individual (EPI's) e, ainda, o alto potencial de absorção desses agrotóxicos, gerando acúmulo nos tecidos, a longo prazo (INCA, 2012; GRABOIS et al., 2014; DE OLIVEIRA, DE ABREU, BARROSO, 2015). Esse fato sugeriu uma correlação entre a expansão dos agrotóxicos no país com o crescente aumento de casos oncológicos, principalmente em agricultores (SILINS, KORHONEN, STENIUS, 2014; INCA, 2015).

A exposição aos agrotóxicos constitui um grave problema de saúde pública no Brasil, especialmente entre trabalhadores rurais, devido aos gastos, longos anos de dedicação ao trabalho e crescente número de óbitos em decorrência da exposição a estes produtos (WHO, 2018). Fatores como monitoramento deficitário da exposição ocupacional aos agrotóxicos, elevadas taxas de subnotificação de casos de intoxicação, a não consideração de 3 determinantes sociais e ambientais na avaliação de riscos químicos e a falta de conhecimento dos trabalhadores rurais quanto ao risco da exposição agravaram esse problema (RIBEIRO et al., 2018). Diante do exposto, considerando que o manejo inadequado dos agrotóxicos e o desconhecimento dos impactos a saúde causados pela exposição são agravantes para o aumento do número de casos de intoxicação por agrotóxicos, o presente estudo propôs reunir dados da literatura para avaliar a relação entre a exposição aos agrotóxicos e o desenvolvimento do processo oncológico, e responder os seguintes questionamentos: 1) Existem evidências científicas que confirmam os impactos negativos do uso dos agrotóxicos para a saúde do ser humano? 2) É possível correlacionar a exposição aos agrotóxicos ao desenvolvimento de neoplasias? 


\section{MATERIAIS E MÉTODOS}

O presente estudo tratou-se de uma revisão sistemática da literatura pautada na exposição aos agrotóxicos como um fator de risco para o desenvolvimento do câncer. Tal metodologia teve como objetivo sintetizar os resultados de diversos estudos primários e buscou responder a uma pergunta de pesquisa claramente formulada. Para tal, utilizou métodos sistemáticos e explícitos para recuperar, selecionar e avaliar os resultados de estudos relevantes. Foi considerada a evidência científica de maior grandeza e foram indicadas na tomada de decisão na prática clínica ou na gestão pública A metodologia utilizada para elaboração da revisão sistemática foi a metodologia PRISMA. A recomendação PRISMA consiste em um checklist com 27 itens e um fluxograma de quatro etapas. O objetivo do PRISMA é ajudar os autores a melhorarem o relato de revisões sistemáticas e meta-análises (GALVAO, PANSANI e HARRAD; 2015).

As buscas foram realizadas no US National Library of Medicine National Institutes of Health (PubMed), instrumento de busca de livre acesso às bases da biblioteca Medical Literature Analysis and Retrieval System Online (Medline) e Literatura Latino-Americana e Caribe em Ciências da Saúde (Lilacs); além do Scientific Electronic Library Online (SciELO), para todos os artigos originais que apontavam alguma associação entre a exposição aos agrotóxicos e o desenvolvimento de neoplasias e os impactos dos agrotóxicos a saúde do homem. A busca foi realizada nos meses de agosto e setembro de 2020. Os filtros da pesquisa foram desenvolvidos de acordo com o dicionário de sinônimos da plataforma - MeSH terms. Para a biblioteca digital PubMed foram utilizados os descritores e operadores booleanos "Cancer AND Agrochemicals", e "Cancer AND Agrochemicals AND Farmworkers". Esses descritores foram adaptados para a base SciELO, os quais também foram utilizados em português. A busca do trabalho restringiu-se aos trabalhos disponíveis em inglês e português e, aos estudos publicados nos últimos 08 anos, a fim de coletar e discutir dados mais recentes sobre o tema.

A seleção inicial foi realizada a partir da leitura dos títulos e resumos, a fim de remover registros que se enquadram nos critérios de exclusão. Os estudos duplicados foram descartados comparando os autores, o título, o ano e o jornal de publicação. Essa primeira análise foi realizada pelo pesquisador (JB) de forma independente e, em seguida, revisada pelos seus pares (PS e LFG). Os critérios de avaliação foram discutidos entre os mesmos e, em caso de dúvida, foi realizado o download dessas publicações para posterior análise e nova discussão entre os pares. Após a 
triagem inicial os estudos potencialmente relevantes foram arquivados em texto completo, para subsequente extração dos dados para elaboração da discussão.

\section{Características do estudo: critérios de inclusão e exclusão}

Como critérios de inclusão foram considerados artigos originais publicados nos últimos 08 anos (2012-2020), com ênfase nos seguintes tópicos: uso de agrotóxicos em humanos, nas lavouras, por agricultores, em estudos de reconhecido rigor científico (revisão sistemática e integrativa; ensaios clínicos; estudos de caso; estudos de coorte; estudo ecológico de série temporal e estudo epidemiológico descritivo) e disponíveis gratuitamente e na íntegra.

Com relação às categorias dos 29 artigos incluídos nesta pesquisa, 17 enquadraram-se no eixo (1) Agrotóxicos e os impactos gerais causados à saúde do homem e, as outras 12 publicações, estavam relacionadas às (2) Neoplasias associadas a exposição aos agrotóxicos. Conforme essas correlações, os estudos que não receberam classificação nas categorias 1 ou 2 foram excluídos por não atenderem à questão norteadora da pesquisa, obtendo-se os resultados aqui apresentados.

A exclusão dos estudos baseou-se em critérios bem definidos, tais como: a) não contemplar os temas da pesquisa; b) data da publicação anterior a 2012; c) pesquisas com experimentação animal; e d) estudos de textos incompletos, livros, editoriais, comentários, cartas ao editor, monografia, dissertações, tese e outros estudos cujos textos completos estavam indisponíveis.

\section{Análise de dados}

Para a análise de dados foram adotadas as diretrizes PRISMA (Reportagem Relativa para Revisão Sistemática e Protocolos de Meta-Análise), as quais englobam discussão entre os autores; observação da revista (qualis); ano de publicação (ordem decrescente); e fatores relevantes para a elaboração da discussão do tema, com análise da exposição ao agrotóxico e o desenvolvimento de tumores.

\section{RESULTADOS E DISCUSSÃO}

Foram encontradas 38 publicações entre artigos, estudos de coorte e resumos, publicados no período de 2012 a 2020. As duplicatas foram retiradas, assim como todos os trabalhos que, após revisão, foram identificados com data de publicação anterior ao período estipulado no 
presente estudo. Após a leitura dos resumos de todas as publicações, restaram 29 artigos atendendo aos critérios de inclusão citados anteriormente. A Figura 01 ilustra os processos de busca e critérios de elegibilidade dos estudos avaliados. No Quadro 01, os artigos elegíveis encontram-se discriminados segundo as características de publicação (autor, ano, periódico e título do trabalho), bem como o desenho e o objetivo do estudo.

Figura 1: Fluxograma com o processo de triagem e de seleção de artigos para a revisão sistemática.

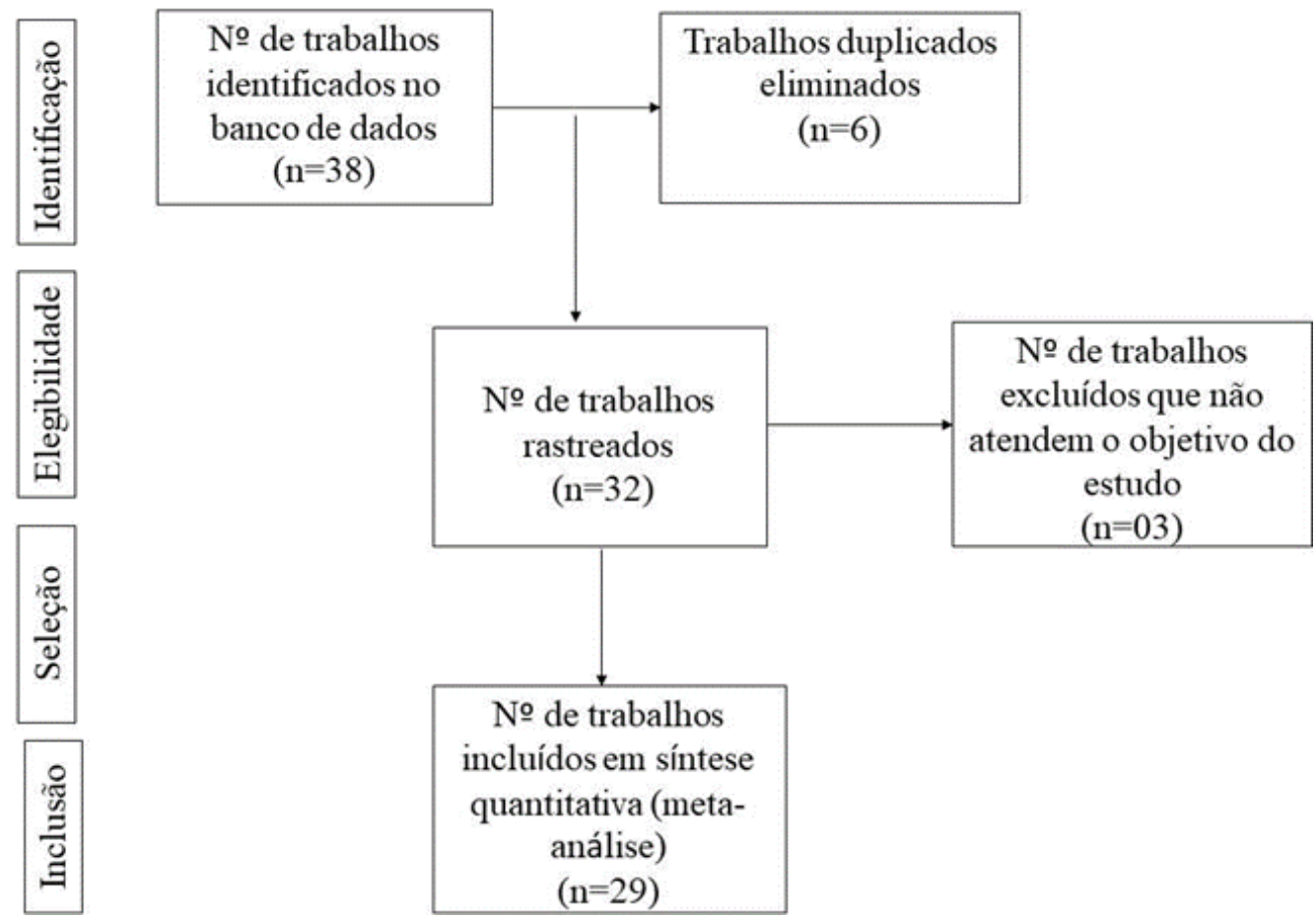

Fonte: Adaptado de Moher et al. (2009) 
Quadro 1: Informações específicas sobre os artigos selecionados de acordo com os critérios de inclusão.

\begin{tabular}{|c|c|c|c|c|}
\hline Autores/Ano & Título & Periódico & Desenho & Objetivo \\
\hline $\begin{array}{l}\text { BOCHNER } e t \\
\text { al., } 2015\end{array}$ & $\begin{array}{l}\text { Óbito ocupacional } \\
\text { por exposição aos } \\
\text { agrotóxicos } \\
\text { utilizado como } \\
\text { evento sentinela: } \\
\text { quando pouco } \\
\text { significa muito }\end{array}$ & $\begin{array}{l}\text { Arca- Repositório } \\
\text { Institucional da } \\
\text { FIOCRUZ }^{3}\end{array}$ & $\begin{array}{l}\text { Estudo de caso } \\
\text { baseado em uma } \\
\text { análise descritiva } \\
\text { de óbitos }\end{array}$ & $\begin{array}{l}\text { Dar subsídios para a } \\
\text { realização de um } \\
\text { modelo de vigilância } \\
\text { sanitária e } \\
\text { epidemiológica, } \\
\text { considerando como } \\
\text { evento sentinela os } \\
\text { óbitos decorrentes de } \\
\text { intoxicações } \\
\text { ocupacionais por } \\
\text { agrotóxicos. }\end{array}$ \\
\hline $\begin{array}{l}\text { CARNEIRO } e t \\
a l ., 2015 \mathrm{a}\end{array}$ & $\begin{array}{l}\text { Os impactos dos } \\
\text { agrotóxicos no } \\
\text { contexto do } \\
\text { agronegócio }\end{array}$ & $\begin{array}{l}\text { Embrapa } \\
\text { Hortaliças- } \\
\text { Capítulo em livro } \\
\text { científico }\end{array}$ & $\begin{array}{l}\text { Revisão } \\
\text { bibliográfica }\end{array}$ & $\begin{array}{l}\text { Apresentar os } \\
\text { impactos dos } \\
\text { agrotóxicos no } \\
\text { contexto do } \\
\text { agronegócio. }\end{array}$ \\
\hline $\begin{array}{l}\text { DE MIRANDA et } \\
\text { al., } 2015\end{array}$ & $\begin{array}{l}\text { Evidências dos } \\
\text { efeitos dos } \\
\text { agrotóxicos na } \\
\text { carcinogênese }\end{array}$ & $\begin{array}{l}\text { Revista da } \\
\text { Graduação - } \\
\text { PUC/RS }\end{array}$ & $\begin{array}{l}\text { Pesquisa } \\
\text { exploratória do } \\
\text { tipo revisão } \\
\text { bibliográfica }\end{array}$ & $\begin{array}{l}\text { Revisar as evidências } \\
\text { dos efeitos dos } \\
\text { agrotóxicos na } \\
\text { carcinogênese. }\end{array}$ \\
\hline $\begin{array}{l}\text { CARNEIRO } \text { et al., } \\
\text { 2015b }\end{array}$ & $\begin{array}{l}\text { Dossiê } \\
\text { Abrasco: um } \\
\text { alerta sobre os } \\
\text { impactos dos } \\
\text { agrotóxicos na } \\
\text { saúde }\end{array}$ & $\begin{array}{l}\text { Arca - Repositório } \\
\text { Institucional da } \\
\text { FIOCRUZ }\end{array}$ & $\begin{array}{l}\text { Estudo de } \\
\text { caso, revisão } \\
\text { bibliográfica }\end{array}$ & $\begin{array}{l}\text { Alertar, por meio de } \\
\text { estudos científicos, as } \\
\text { autoridades públicas } \\
\text { nacionais, } \\
\text { internacionais e a } \\
\text { sociedade em geral } \\
\text { para a necessidade de } \\
\text { políticas que possam } \\
\text { proteger e promover } \\
\text { a saúde humana e os } \\
\text { ecossistemas. }\end{array}$ \\
\hline $\begin{array}{l}\text { CEZAR-VAZ et al., } \\
2016\end{array}$ & $\begin{array}{l}\text { Sociedade de } \\
\text { risco: o uso } \\
\text { dos } \\
\text { agrotóxicos e } \\
\text { implicações na } \\
\text { saúde do } \\
\text { trabalhador } \\
\text { rural }\end{array}$ & Escola Anna Nery & $\begin{array}{l}\text { Estudo } \\
\text { quantitativo, } \\
\text { transversal, } \\
\text { observacional } \\
\text { e exploratório }\end{array}$ & $\begin{array}{l}\text { Aplicar uma } \\
\text { abordagem } \\
\text { socioambiental na } \\
\text { relação entre saúde } \\
\text { humana e trabalho } \\
\text { rural por meio da } \\
\text { verificação de da } \\
\text { associação entre os } \\
\text { distúrbios de saúde e } \\
\text { o uso de agrotóxicos. }\end{array}$ \\
\hline
\end{tabular}


Continuação do Quadro 1

\begin{tabular}{|c|c|c|c|c|}
\hline $\begin{array}{l}\text { RIGOTTO, } \\
\text { AGUIAR, } 2016\end{array}$ & $\begin{array}{c}\text { Invisibilidade ou } \\
\text { invisibilização dos } \\
\text { efeitos crônicos } \\
\text { dos agrotóxicos à } \\
\text { saúde? Desafios à } \\
\text { ciência e às } \\
\text { políticas públicas }\end{array}$ & $\begin{array}{c}\text { Observatório } \\
\text { Internacional de } \\
\text { Capacidades } \\
\text { Humanas }\end{array}$ & $\begin{array}{l}\text { Revisão } \\
\text { bibliográfica }\end{array}$ & $\begin{array}{l}\text { Dialogar com } \\
\text { questões que } \\
\text { contribuem para } \\
\text { compreender a } \\
\text { construção } \\
\text { social da } \\
\text { invisibilidade } \\
\text { dos efeitos } \\
\text { crônicos da } \\
\text { exposição aos } \\
\text { agrotóxicos. }\end{array}$ \\
\hline BA et al., 2016 & $\begin{array}{l}\text { Occupational } \\
\text { exposure to } \\
\text { pesticides and } \\
\text { other biocides and } \\
\text { risk of thyroid } \\
\text { cancer }\end{array}$ & $\begin{array}{l}\text { Occupational } \\
\text { and } \\
\text { environmental } \\
\text { medicine }\end{array}$ & $\begin{array}{l}\text { Estudo de caso- } \\
\text { controle de } \\
\text { base } \\
\text { populacional }\end{array}$ & $\begin{array}{l}\text { Explorar as } \\
\text { associações entre } \\
\text { exposição } \\
\text { ocupacional a } \\
\text { agrotóxicos e o risco } \\
\text { de } \mathrm{CA}^{2} \text { de tireoide }\end{array}$ \\
\hline $\begin{array}{l}\text { LOPES, } \\
\text { ALBUQUERQUE, } \\
2018\end{array}$ & $\begin{array}{l}\text { Agrotóxicos e } \\
\text { seus impactos na } \\
\text { saúde humana e } \\
\text { ambiental: uma } \\
\text { revisão } \\
\text { sistemática }\end{array}$ & Saúde em debate & $\begin{array}{l}\text { Revisão } \\
\text { sistemática } \\
\text { (Metodologia } \\
\text { PRISMA) }\end{array}$ & $\begin{array}{l}\text { Conhecer os rumos } \\
\text { da investigação } \\
\text { acerca do uso de } \\
\text { agrotóxicos e sua } \\
\text { relação com a saúde }\end{array}$ \\
\hline $\begin{array}{l}\text { GROSICKA- } \\
\text { MACIAG } e t \\
\text { al., } 2013\end{array}$ & $\begin{array}{l}\text { Modulation of } \\
\text { antioxidant } \\
\text { defense system } \\
\text { by the } \\
\text { dithiocarbamate } \\
\text { fungicides Maneb } \\
\text { and Zineb in } \\
\text { Chinese hamster } \\
\text { V79 cells and the } \\
\text { role of N-acetyl- } \\
\text { L-cysteine }\end{array}$ & $\begin{array}{l}\text { Food and } \\
\text { chemical } \\
\text { toxicology }\end{array}$ & $\begin{array}{l}\text { Estudo in } \\
\text { vitro }\end{array}$ & $\begin{array}{l}\text { Examinar a } \\
\text { potência de } \\
\text { agrotóxicos para } \\
\text { induzir alterações } \\
\text { nas atividades de } \\
\text { enzimas } \\
\text { antioxidantes em } \\
\text { células de } \\
\text { fibroblastos }\end{array}$ \\
\hline
\end{tabular}


Continuação do Quadro 1

\begin{tabular}{|c|c|c|c|c|}
\hline $\begin{array}{l}\text { MIRANDA } e t \\
a l ., 2016\end{array}$ & $\begin{array}{l}\text { A utilização de } \\
\text { agrotóxicos nos } \\
\text { municípios de } \\
\text { Jataí e Perolândia } \\
\left(\mathrm{GO}^{5}\right) \text { : } \\
\text { embasamento } \\
\text { legal e impacto na } \\
\text { saúde pública }\end{array}$ & $\begin{array}{l}\text { Repositório } \\
\text { Digital de Teses e } \\
\text { Dissertações - } \\
\text { Universidade } \\
\text { Federal de Goiás }\end{array}$ & $\begin{array}{l}\text { Pesquisa de } \\
\text { campo qualitativa }\end{array}$ & $\begin{array}{l}\text { Verificar como é } \\
\text { feito o uso de } \\
\text { agrotóxicos no } \\
\text { Município de Jatai e } \\
\text { no Assentamento } \\
\text { Três Pontes } \\
\text { Perolândia }\left(\mathrm{GO}^{5}\right) \text { e } \\
\text { analisar o } \\
\text { cumprimento da } \\
\text { legislação vigente em } \\
\text { relação à proteção e } \\
\text { prevenção dos riscos } \\
\text { à saúde }\end{array}$ \\
\hline $\begin{array}{l}\text { TEYSSEIRE } \text { et } \\
\text { al., } 2020\end{array}$ & $\begin{array}{l}\text { Assessment of } \\
\text { residential } \\
\text { exposures to } \\
\text { agricultural } \\
\text { pesticides: A } \\
\text { scoping review }\end{array}$ & Plos one & $\begin{array}{l}\text { Revisão } \\
\text { sistemática } \\
\text { (Metodologia } \\
\text { PRISMA) }\end{array}$ & $\begin{array}{l}\text { Caracterizar a } \\
\text { exposição residencial } \\
\text { a pesticidas agrícolas } \\
\text { e identificar } \\
\text { potenciais lacunas } \\
\text { nesta área de } \\
\text { pesquisa }\end{array}$ \\
\hline OMS, 2019 & Cancer & $\begin{array}{l}\text { World Health } \\
\text { Organization }\end{array}$ & N.A ${ }^{1}$ & N. $A^{1}$ \\
\hline $\begin{array}{l}\text { GONÇALVES } \\
\text { et al., } 2012\end{array}$ & $\begin{array}{l}\text { Uso de } \\
\text { agrotóxicos e a } \\
\text { relação com a } \\
\text { saúde na etnia } \\
\text { Xukuru do } \\
\text { Ororubá, } \\
\text { Pernambuco, } \\
\text { Brasil }\end{array}$ & Saúde e Sociedade & $\begin{array}{l}\text { Estudo de } \\
\text { caso }\end{array}$ & $\begin{array}{l}\text { Compreender o } \\
\text { processo de } \\
\text { utilização de } \\
\text { agrotóxicos no } \\
\text { cultivo agrícola e a } \\
\text { relação com a saúde } \\
\text { indígena }\end{array}$ \\
\hline
\end{tabular}




\section{Continuação do Quadro 1}

\begin{tabular}{|c|c|c|c|c|}
\hline $\begin{array}{l}\text { KAHL, SILVA, } \\
2016\end{array}$ & $\begin{array}{l}\text { Telomere } \\
\text { measurement in } \\
\text { individuals } \\
\text { occupationally } \\
\text { exposed to } \\
\text { pesticide mixtures } \\
\text { in tobacco fields }\end{array}$ & $\begin{array}{l}\text { Environmental } \\
\text { and molecular } \\
\text { mutagenesis }\end{array}$ & $\begin{array}{l}\text { Abordagem de } \\
\text { sistema biológico }\end{array}$ & $\begin{array}{l}\text { Avaliar a interação } \\
\text { entre a mistura de } \\
\text { pesticidas para cultivo } \\
\text { de tabaco, bem como } \\
\text { os processos } \\
\text { biológicos } \\
\text { relacionados a essa } \\
\text { exposição, a fim de } \\
\text { fornecer informações } \\
\text { sobre a influência } \\
\text { dessa exposição e } \\
\text { alterações nas } \\
\text { atividades de } \\
\text { telômeros }\end{array}$ \\
\hline $\begin{array}{l}\text { CARGNIN, } \\
\text { ECHER e SILVA, } \\
2017\end{array}$ & $\begin{array}{l}\text { Fumicultura: uso } \\
\text { de equipamento } \\
\text { de proteção } \\
\text { individual e } \\
\text { intoxicação por } \\
\text { agrotóxico }\end{array}$ & $\begin{array}{l}\text { Revista de } \\
\text { Pesquisa: Cuidado } \\
\text { é Fundamental } \\
\text { Online }\end{array}$ & Estudo transversal & $\begin{array}{l}\text { Verificar o uso de } \\
\text { EPI }^{7} \text { e a presença de } \\
\text { sintomas de } \\
\text { intoxicação por } \\
\text { agrotóxicos em } \\
\text { fumicultores }\end{array}$ \\
\hline $\begin{array}{l}\text { FREIRE, } \\
\text { KOIFMAN, } 2017\end{array}$ & $\begin{array}{l}\text { Occupational } \\
\text { exposure to } \\
\text { pesticides, } \\
\text { reproductive } \\
\text { hormone levels } \\
\text { and sperm } \\
\text { quality in young } \\
\text { Brazilian men }\end{array}$ & $\begin{array}{l}\text { Reproductive } \\
\text { Toxicology }\end{array}$ & $\begin{array}{l}\text { Pesquisa de } \\
\text { biomonitoramento }\end{array}$ & $\begin{array}{l}\text { Investigar a } \\
\text { distribuição dos } \\
\text { níveis séricos de } \\
\text { pesticidas } \\
\text { organoclorados em } \\
\text { doadores de sangue } \\
\text { brasileiros e } \\
\text { determinar os fatores } \\
\text { que podem } \\
\text { influenciar os níveis } \\
\text { de exposição }\end{array}$ \\
\hline $\begin{array}{l}\text { SILVA et al., } \\
2013\end{array}$ & $\begin{array}{l}\text { Prevalência de } \\
\text { morbidades e } \\
\text { sintomas em } \\
\text { idosos: um } \\
\text { estudo } \\
\text { comparativo } \\
\text { entre zonas rural } \\
\text { e urbana }\end{array}$ & $\begin{array}{l}\text { Ciência e saúde } \\
\text { coletiva }\end{array}$ & Estudo transversal & $\begin{array}{l}\text { Caracterizar a } \\
\text { população de idosos } \\
\text { que reside no } \\
\text { município de } \\
\text { Cachoeira do Sul } \\
\left(\mathrm{RS}^{8}\right) \text {, investigando } \\
\text { morbidades e } \\
\text { sintomas referidos } \\
\text { segundo zona de } \\
\text { moradia rural/urbana } \\
\text { e uso de agrotóxicos }\end{array}$ \\
\hline $\begin{array}{l}\text { GANGEMI et al., } \\
2016\end{array}$ & $\begin{array}{l}\text { Occupational } \\
\text { exposure to } \\
\text { pesticides as a } \\
\text { possible risk } \\
\text { factor for the } \\
\text { development of } \\
\text { chronic diseases } \\
\text { in humans }\end{array}$ & $\begin{array}{l}\text { Molecular } \\
\text { medicine reports }\end{array}$ & $\begin{array}{l}\text { Revisão } \\
\text { bibliográfica }\end{array}$ & $\begin{array}{l}\text { Avaliar o impacto } \\
\text { dos agrotóxicos na } \\
\text { saúde humana, } \\
\text { principalmente no } \\
\text { que se refere ao } \\
\text { desenvolvimento de } \\
\text { doenças crônicas }\end{array}$ \\
\hline
\end{tabular}




\section{Continuação do Quadro 1}

\begin{tabular}{|c|c|c|c|c|}
\hline PLUTH, 2017 & $\begin{array}{l}\text { Exposição aos } \\
\text { agrotóxicos e } \\
\text { câncer }\end{array}$ & $\begin{array}{l}\text { Repositório } \\
\text { Digital da } \\
\text { Universidade } \\
\text { Federal da } \\
\text { Fronteira do } \\
\text { Sul }\end{array}$ & $\begin{array}{l}\text { Revisão } \\
\text { bibliográfica }\end{array}$ & $\begin{array}{l}\text { Revisar trabalhos já } \\
\text { publicados que } \\
\text { investigaram a } \\
\text { relação câncer e } \\
\text { agrotóxico e } \\
\text { descrever o perfil } \\
\text { epidemiológico dos } \\
\text { casos de } \mathrm{CA}^{2} \text { de um } \\
\text { Centro de Alta } \\
\text { Complexidade em } \\
\text { Oncologia } \\
\left(\text { CACON }{ }^{9}\right) \text {, bem } \\
\text { como investigar a } \\
\text { relação entre } \\
\text { morbidade por } \mathrm{CA}^{2} \mathrm{e} \\
\text { exposição aos } \\
\text { agrotóxicos }\end{array}$ \\
\hline BORGES, 2018 & $\begin{array}{l}\text { O processo de } \\
\text { institucionalização } \\
\text { do uso de } \\
\text { agrotóxicos entre } \\
\text { pequenos } \\
\text { cotonicultores } \\
\text { brasileiros }\end{array}$ & $\begin{array}{l}\text { Repositório } \\
\text { Digital } \\
\text { Universidade } \\
\text { Federal de } \\
\text { Minas Gerais }\end{array}$ & $\begin{array}{l}\text { Estudo de casos } \\
\text { múltiplos sob uma } \\
\text { perspectiva } \\
\text { longitudinal }\end{array}$ & $\begin{array}{l}\text { Analisar o processo } \\
\text { de institucionalização } \\
\text { do uso de agrotóxicos } \\
\text { entre pequenos } \\
\text { cotonicultores } \\
\text { brasileiros de } 1945 \text { a } \\
2018\end{array}$ \\
\hline $\begin{array}{l}\text { GRANGEIRO } \\
\text { et al., } 2019\end{array}$ & $\begin{array}{l}\text { Incidência e } \\
\text { mortalidade por } \\
\text { câncer de próstata } \\
\text { no Tocantins e } \\
\text { Palmas, no período } \\
\text { de } 2010 \text { a } 2014\end{array}$ & $\begin{array}{l}\text { Revista de } \\
\text { Patologia } \\
\text { do } \\
\text { Tocantins }\end{array}$ & $\begin{array}{l}\text { Estudo } \\
\text { retrospectivo }\end{array}$ & $\begin{array}{l}\text { Realizar um } \\
\text { levantamento acerca } \\
\text { da incidência e } \\
\text { mortalidade por } \mathrm{CA}^{2} \\
\text { de próstata no Estado } \\
\text { do Tocantins e na } \\
\text { cidade de Palmas, no } \\
\text { período entre } 2010 \text { e } \\
2014\end{array}$ \\
\hline $\begin{array}{l}\text { CHA et al., } \\
2014\end{array}$ & $\begin{array}{l}\text { Trends in pesticide } \\
\text { suicide in South } \\
\text { Korea, 1983-2014 }\end{array}$ & $\begin{array}{l}\text { Epidemiol } \\
\text { ogy and } \\
\text { psychiatri } \\
\text { c sciences }\end{array}$ & $\begin{array}{l}\text { Revisão } \\
\text { bibliográfica } \\
\text { crítica }\end{array}$ & $\begin{array}{l}\text { Revisar o uso e as } \\
\text { tendências de } \\
\text { pesticidas agrícolas e } \\
\text { identificar os } \\
\text { perigosos para } \\
\text { regulamentação, em } \\
\text { termos de saúde } \\
\text { pública, na Coreia do } \\
\text { Sul }\end{array}$ \\
\hline
\end{tabular}




\section{Continuação do Quadro 1}

\begin{tabular}{|c|c|c|c|c|}
\hline PLUTH, 2019 & $\begin{array}{l}\text { Pesticide } \\
\text { exposure and } \\
\text { cancer: an } \\
\text { integrative } \\
\text { literature review }\end{array}$ & Saúde em Debate & $\begin{array}{l}\text { Revisão } \\
\text { integrativa }\end{array}$ & $\begin{array}{l}\text { Realizar uma revisão } \\
\text { integrativa da } \\
\text { literatura de estudos } \\
\text { publicados sobre } \\
\text { exposição aos } \\
\text { agrotóxicos e } \mathrm{CA}^{2} \\
\text { com foco em } \\
\text { agricultores } \\
\text { considerando todos } \\
\text { os tipos de } \mathrm{CA}^{2} \text {, } \\
\text { agrotóxicos e faixas } \\
\text { etárias }\end{array}$ \\
\hline LEON et al., 2019 & $\begin{array}{l}\text { Pesticide use and } \\
\text { risk of non- } \\
\text { Hodgkin } \\
\text { lymphoid } \\
\text { malignancies in } \\
\text { agricultural } \\
\text { cohorts from } \\
\text { France, Norway } \\
\text { and the USA: a } \\
\text { pooled analysis } \\
\text { from the } \\
\text { AGRICOH }{ }^{10} \\
\text { consortium }\end{array}$ & $\begin{array}{l}\text { International } \\
\text { Journal of } \\
\text { Epidemiology }\end{array}$ & Estudo de coorte & $\begin{array}{l}\text { Investigaram a } \\
\text { relação do uso de } \\
\text { pesticidas } \\
\text { selecionados com } \\
\text { malignidades } \\
\text { linfoides não } \\
\text { Hodgkin }\left(\mathrm{LNH}^{11}\right)\end{array}$ \\
\hline SMITH et al., 2017 & $\begin{array}{l}2,4- \\
\text { Dichlorophenoxy } \\
\text { acetic acid (2,4- } \\
\text { D) and risk of } \\
\text { non-Hodgkin } \\
\text { lymphoma: a } \\
\text { meta-analysis } \\
\text { accounting for } \\
\text { exposure levels }\end{array}$ & $\begin{array}{l}\text { Annals of } \\
\text { Epidemiology }\end{array}$ & $\begin{array}{l}\text { Revisão } \\
\text { sistemática e } \\
\text { meta-análise dos } \\
\text { estudos } \\
\text { epidemiológicos } \\
\text { (Metodologia } \\
\text { PRISMA) }\end{array}$ & $\begin{array}{l}\text { Verificar através de } \\
\text { meta-análise se há } \\
\text { uma associação entre } \\
\text { a exposição ao 2,4-D } \\
\text { e o LNH }\end{array}$ \\
\hline $\begin{array}{l}\text { LEMARCHAND et } \\
\text { al., } 2016\end{array}$ & $\begin{array}{l}\text { Prostate cancer } \\
\text { risk among } \\
\text { French farmers in } \\
\text { the AGRICAN }{ }^{12} \\
\text { cohort }\end{array}$ & $\begin{array}{l}\text { Scandinavian } \\
\text { Journal of } \\
\text { work, } \\
\text { environment } \\
\text { \& health }\end{array}$ & $\begin{array}{l}\text { Estudo de } \\
\text { coorte } \\
\text { prospectivo }\end{array}$ & $\begin{array}{l}\text { Identificar fatores de } \\
\text { risco ocupacionais } \\
\text { para } \mathrm{CA}^{2} \text { de próstata } \\
\text { entre agricultores no } \\
\text { estudo de } \\
\quad \text { coorte } \\
\text { prospectivo } \\
\text { AGRICAN }{ }^{12}\end{array}$ \\
\hline
\end{tabular}




\section{Continuação do Quadro 1}

\begin{tabular}{|c|c|c|c|c|}
\hline SILVA et al., 2015 & $\begin{array}{l}\text { Correlação entre } \\
\text { produção } \\
\text { agrícola, variáveis } \\
\text { clínicas- } \\
\text { demográficas e } \\
\mathrm{CA}^{2} \text { de próstata: } \\
\text { um estudo } \\
\text { ecológico }\end{array}$ & $\begin{array}{l}\text { Ciência e Saúde } \\
\text { Coletiva }\end{array}$ & $\begin{array}{l}\text { Estudo ecológico, } \\
\text { de caráter } \\
\text { exploratório }\end{array}$ & $\begin{array}{l}\text { Explorar correlações } \\
\text { entre variáveis } \\
\text { relativas à produção } \\
\text { agrícola, ao uso de } \\
\text { serviços de saúde e } \\
\text { sociodemográficas e } \\
\text { às taxas de } \\
\text { mortalidade por } \mathrm{CA}^{2} \\
\text { de próstata entre } \\
\text { 2005-2009, nos } \\
\text { estados brasileiros }\end{array}$ \\
\hline ENGEL et al., 2017 & $\begin{array}{l}\text { Insecticide use } \\
\text { and breast cancer } \\
\text { risk among } \\
\text { farmers' wives in } \\
\text { the Agricultural } \\
\text { Health Study }\end{array}$ & $\begin{array}{l}\text { Environmental } \\
\text { health } \\
\text { perspectives }\end{array}$ & $\begin{array}{l}\text { Estudo de coorte } \\
\text { prospectivo }\end{array}$ & $\begin{array}{l}\text { Examinar a associação } \\
\text { entre ouso de } \\
\text { pesticidas e a } \\
\text { incidência de } \mathrm{CA}^{2} \text { de } \\
\text { mama }\end{array}$ \\
\hline LERRO et al., 2017 & $\begin{array}{l}\text { A longitudinal } \\
\text { study of atrazine } \\
\text { and } 2,4-\mathrm{D} \\
\text { exposure and } \\
\text { oxidative stress } \\
\text { markers among } \\
\text { Iowa corn farmers }\end{array}$ & $\begin{array}{l}\text { Environmental } \\
\text { and molecular } \\
\text { mutagenesis }\end{array}$ & $\begin{array}{l}\text { Estudo } \\
\text { longitudinal }\end{array}$ & $\begin{array}{l}\text { Examinar o impacto } \\
\text { da exposição aos } \\
\text { herbicidas } \\
\text { amplamente usados } \\
\text { atrazina e ácido 2,4- } \\
\text { diclorofenoxiacético } \\
\text { (2,4-D) em } \\
\text { marcadores de } \\
\text { estresse oxidativo }\end{array}$ \\
\hline
\end{tabular}

Fonte: Autores, 2021.

Legenda: 1. NA: Não se Aplica - Estudos que não consideraram uma amostra específica. 2. CA: Câncer. 3. FIOCRUZ: Fundação Oswaldo Cruz. 4. PUC/RS: Pontifícia Universidade Católica/Rio Grande do Sul. 5. GO: Goiás. 6. OMS: Organização Mundial da Saúde. 07. EPI: Equipamento de Proteção Individual. 8. RS: Rio Grande do Sul. 9. CACON: Centro de Alta Complexidade em Oncologia. 10. AGRICOH: A Consertium of Agricultural Cohort Studies. 11. LNH: Linfoma Não Hodking. 12. AGRICAN: Agriculture and Cancer. 
Para melhor exposição dos dados e suas respectivas discussões, o primeiro tópico apontou os principais trabalhos publicados nos últimos oito anos (2012-2020), os quais indicaram diferentes impactos possivelmente causados pela exposição aos agrotóxicos na saúde humana. Posteriormente, no segundo tópico, foram discutidos os estudos que analisaram a ocorrência de câncer em populações expostas a agrotóxicos. Mesmo considerando as neoplasias como um dos impactos da exposição aos agrotóxicos, optou-se por discutir esse assunto em um tópico isolado em detrimento da relevância do tema. Portanto, foram considerados os principais tipos de neoplasias já apontados pela literatura que têm a exposição aos agrotóxico como um potencial fator de risco (neoplasia de mama, próstata, tireoide, linfoma) (PERTILE et al., 2018; INCA, 2016; RIGOTTO, AGUIAR, 2016).

\section{i) Agrotóxicos e os impactos gerais a saúde do homem}

Atualmente, os vários impactos à saúde relacionados a exposição aos agrotóxicos já são conhecidos (BOCHNER et al., 2015; CARNEIRO, 2015a; CEZAR-VAZ et al.,2016). Os de maior relevância para a saúde humana e ambiental são as poluições e/ou contaminações e as intoxicações agudas e crônicas (CARNEIRO et al., 2015b; RIGOTTO, AGUIAR, 2016). Há riscos para o homem quando o agrotóxico entra em contato direto com o ser humano por manipulação inadequada ou, indiretamente, através da ingestão de alimentos e de água (LOPES, ALBUQUERQUE, 2018).

Grosicka-Maciag (2013) avaliou diversos trabalhos que apontaram que a ação tóxica dos agrotóxicos pode induzir o estresse oxidativo e o acúmulo de radicais livres na célula. $\mathrm{O}$ estresse oxidativo agudo ou de longa duração perturba o metabolismo celular e é capaz de produzir mudanças permanentes na estrutura das proteínas, lipídios e DNA, resultando em perda ou aumento da atividade como resultado do estresse oxidativo. De acordo com o autor, esse evento pode acelerar o desenvolvimento de várias doenças, processos neurodegenerativos que resultam em doenças como Alzheimer e Parkinson.

Envolvendo a mesma temática, Teysseyre et al. (2020) realizaram uma revisão sistemática a fim de caracterizar a exposição residencial a pesticidas agrícolas e identificar potenciais lacunas nesta área de pesquisa. Após a análise de diversos trabalhos, foram encontrados 98 estudos epidemiológicos que investigavam possíveis ligações entre a exposição aos pesticidas e o início de efeitos adversos à saúde, incluindo infertilidade e má 
formação congênita de fetos.

Com objetivo de compreender como esse processo de utilização de agrotóxicos no cultivo agrícola pode impactar na saúde da população, um estudo de caso realizado entre os índios Xukuru do Ororubá, em Pesqueira, Pernambuco-Brasil (GONÇALVES et al., 2012) mostrou que o uso dos produtos químicos ocorreu de forma intensiva e sem a utilização de equipamento de proteção individual nessa população. Além disso, 10\% dos indígenas relataram já ter sofrido intoxicação por agrotóxicos e 45,5\% referiram sentir fortes dores de cabeça e tonturas imediatamente após a aplicação dos produtos, o que demonstrou o impacto do uso intenso desses produtos a saúde da etnia indígena em questão.

Assim como a população indígena, trabalhadores rurais são grupos populacionais potencialmente expostos aos agrotóxicos. Lopes e Albuquerque (2018), em uma revisão sistemática, avaliaram pesquisas que abordaram os riscos de intoxicação por agrotóxicos nesses trabalhadores. Estudos com cultivadores de tabaco que manipulavam os agrotóxicos mostraram que essa população apresentou danos nos mecanismos de defesa celular e alterações nas atividades de telômeros, além de transtornos mentais e outras doenças ocupacionais (KAHL, SILVA, 2016). Outro grupo de trabalhador rural estudado foi o dos fumicultores, os quais utilizavam pesticidas nas suas atividades. Segundo as entrevistas do estudo de Cargnin, Echer e Silva (2017), alguns desses profissionais relataram sintomas como dores de cabeça, náuseas e dor de estômago, além de dor lombar, disúria e diagnóstico médico de gastrite/epigastralgia, depressão, ansiedade, mialgia, irritabilidade e cólicas abdominais. Os estudos apontaram que os sintomas relatados pelos trabalhadores podem estar associados ao uso dos agrotóxicos em suas atividades laborais.

Freire, Koifman (2017) avaliaram, especificamente, os efeitos dos organoclorados na saúde do homem e identificaram que esses compostos podem exercer efeitos adversos no tecido hematopoiético e no fígado em populações cronicamente expostas a níveis elevados. Os organoclorados também foram relacionados a alterações nas concentrações hormonais, assim como outros agrotóxicos foram associados a alterações nos sistemas reprodutores masculinos e femininos.

Outras entrevistas realizadas com populações residentes em áreas de uso intensivo de agroquímicos corroboraram os dados dos estudos citados anteriormente. Os entrevistados referiram doenças e sintomas como diabetes, doença de Alzheimer, boca seca, visão alterada, dor nas pernas, doenças neurológicas, síndromes dolorosas e doenças orais (SILVA et al., 2013). 
Com o objetivo de avaliar se os agrotóxicos têm relação com o desenvolvimento de doenças crônicas, como as citadas acima, Gangemi et al. (2016) realizaram uma revisão bibliográfica sobre o tema e, embora existam muitas pistas que apontem para a associação entre

os pesticidas e a incidência de doenças como diabetes mellitus ou câncer, os estudos experimentais e as revisões sistemáticas sobre o assunto foram inconclusivos.

\section{ii) Neoplasias associadas a exposição aos agrotóxicos}

Vários estudos têm mostrado a associação positiva entre exposição aos agrotóxicos e doenças como diversos tipos de neoplasias (DE MIRANDA et al., 2015; PLUTH, 2017). Em março de 2015, por exemplo, a justiça Norte Americana considerou o herbicida glifosato, comercialmente conhecido como Roundup ${ }^{\circledR}$, como "um fator substancial" para o desenvolvimento de linfoma não-Hodgink em um homem de 70 anos de idade morador do norte de São Francisco (Califórnia), que atribui a doença ao uso continuado do herbicida, um dos mais populares do mundo. Segundo dados compilados pelo Ibama e Ministério da Agricultura, o glifosato é o agrotóxico mais comercializado no Brasil (BORGES et al., 2018) e, apesar de reguladores europeus e norte-americanos afirmarem que não há comprovação de que esse composto químico cause tumores malignos, a OMS (2019), no entanto, ressaltou a probabilidade de o composto ser carcinogênico (GRANGEIRO et al., 2019).

O alerta da OMS veio de encontro a alguns trabalhos já publicados sobre o assunto, como em uma revisão crítica sobre o potencial cancerígeno de pesticidas usados na Coreia. Pesquisadores verificaram que os pesticidas foram responsáveis por 24\% (5.856 / 24.795 toneladas) do volume total de consumo no país com destaque para o fato de que $31 \%$ dos 987 pesticidas comercializados continham ingredientes cancerígenos para humanos. Curiosamente, os produtos considerados com menor toxicidade apresentaram maior potencial carcinogênico no referido estudo (CHA et al., 2014).

Assim como na Coréia, outros países também investigaram fatores associados a incidência de câncer. No Canadá, um estudo de base populacional com trabalhadores agrícolas apontou que 9.515 casos de neoplasias malignas diagnosticadas no país ocorreram em trabalhadores agrícolas. Entre os homens, foram observados riscos aumentados para linfoma não Hodgkin, melanoma, tumor de próstata e de lábio, e entre as trabalhadoras 
agrícolas para neoplasia de pâncreas. Riscos aumentados de melanoma, leucemia e mieloma múltiplo também foram observados em um subconjunto de agricultoras. O estudo sugeriu que a exposição aos pesticidas pode ter contribuído para o aumento dos riscos de câncer hematopoiético, entretanto o aumento do risco de neoplasia de lábio e melanoma pode ser atribuído à exposição ao sol e não aos pesticidas (PLUTH, ZANINI e BATTISTI, 2019).

A maioria das análises realizadas por Leon et al. (2019) também não conseguiu sugerir associação entre a ocorrência de linfomas e os agrotóxicos. Os autores investigaram à relação do uso de 14 grupos químicos de pesticidas selecionados e 33 ingredientes químicos ativos individuais com malignidades linfoides não Hodgkin (LNH) gerais ou subtipos principais, em uma análise conjunta de três grandes coortes de trabalhadores agrícolas. Durante o acompanhamento, 2.430 casos de LNH foram diagnosticados em 316.270 agricultores, acumulando 3.5-74.815 pessoas-ano sob risco, entretanto, a maior parte das análises não sugeriu associação positiva. Em contrapartida, uma revisão sistemática de vários estudos epidemiológicos já havia encontrado fortes evidências para uma associação entre LNH e exposição ao herbicida 2,4-D (SMITH et al., 2017). Um total de 12 estudos observacionais, 11 estudos de caso-controle e um estudo de coorte foram incluídos. $\mathrm{O}$ risco relativo resumido para LNH usando resultados de estudos comparando indivíduos que nunca foram expostos ao 2,4-D foi de 1,38 (intervalo de confiança de 95\% (IC), 1,071,77). No entanto, em análises com foco em resultados de grupos altamente expostos, o risco relativo resumido para $\mathrm{LNH}$ foi de 1,73 (IC de 95\%, 1,10-2,72).

Com o objetivo de identificar fatores de risco ocupacionais para tumor de próstata entre agricultores, Lemarchand et al. (2016), realizaram um estudo de coorte. O uso de pesticidas pelos produtores de frutas foi associado a um risco elevado de neoplasia de próstata, assim como para produtores de batata e tabaco, uma vez que todas essas atividades envolviam uso de pesticidas. Com a mesma proposta, Silva et al. (2015) fizeram uma análise univariada de dados espaciais para investigação da autocorrelação espacial global nas taxas de mortalidade por CA de próstata nos estados brasileiros entre 2005 e 2009. Examinou-se a correlação entre indicadores sociodemográficos, dados de produção agrícola, variáveis relacionadas ao uso de serviços de saúde, consumo alimentar e taxas de mortalidade por neoplasia de próstata. Diante dos resultados apurados, identificou-se correlação positiva entre toneladas de soja plantadas e a mortalidade pela doença, sugerindo a possível existência de associação entre os agrotóxicos utilizados no plantio da soja e neoplasias. 
Em relação a exposição de mulheres aos agroquímicos, Engel et al. (2017) examinaram a incidência de neoplasia de mama naquelas que trabalhavam com esse tipo de produto, através de um estudo prospectivo de Saúde Agrícola. Durante um seguimento médio de 14,7 anos, 39\% das mulheres relataram usar inseticidas e 1.081 foram diagnosticadas com neoplasia de mama, o que possibilitou a associação do uso dos organofosforados a um risco elevado de ocorrência da doença. Um estudo publicado em 2013 (Silva et al., 2013), de caso controle com a população feminina residente na cidade de Rondonópolis, sul do estado de Mato Grosso, também avaliou o uso de agrotóxicos e a ocorrência de tumores malignos na mama nessa população. O estudo identificou que mulheres que moravam perto de terras agrícolas com pesticidas (OR: 2,37; IC: 95\% 1,783,16) e tinham idade superior a 50 anos e menarca precoce (OR: 2,08; IC: 95\% 1,06-4,12), tiveram um maior risco de desenvolver câncer de mama em comparação aos controles. Os resultados encontrados nesses estudos apontaram que os agrotóxicos pareceram ser um fator de risco ambiental para o desenvolvimento de neoplasias de mama em mulheres.

Além desses, outros tipos de canceres também foram estudados como resultado da exposição aos agrotóxicos. Dados de um estudo caso-controle de base populacional envolvendo 462 casos de neoplasia de tireoide e 498 controles em Connecticut coletados em 2010-2011, Ba et al. (2016) examinaram a relação entre esses diagnósticos com a exposição ocupacional a biocidas e pesticidas por meio de uma matriz de exposição no trabalho. Indivíduos que já foram ocupacionalmente expostos a biocidas tiveram um risco aumentado de desenvolver a doença (Odds Ratio = 1,65; Intervalo de Confiança de 95\% 1,16 a 2,35), e o maior risco foi observado para a alta probabilidade cumulativa de exposição (Odds Ratio $=2,18 ; 95 \%$ Intervalo de Confiança 1,28 a 3,73).

Associações semelhantes foram observadas em relação a outros tipos de neoplasias de tireoide. O estudo de caso-controle de Lerro et al. (2017) também avaliou a associação de pesticidas organoclorados e tumores de tireoide. Após as análises realizadas, o estudo identificou que as emissões de pesticidas organoclorados variaram ao longo do tempo, sugerindo, portanto, que diferentes padrões de risco por coorte de nascimento acusam a importância potencial do momento da exposição em relação ao desenvolvimento da doença. Os pesquisadores destacaram que é imprescindível uma avaliação adicional dessas associações para estabelecer uma associação mais precisa diante da natureza multifatorial associada ao desenvolvimento de tumores. 


\section{CONCLUSÃO}

Diante desses dados, foi possível observar um importante papel dos estudos epidemiológicos no processo de avaliação de carcinogenicidade dos agrotóxicos. Ser agricultor e estar potencialmente exposto a agrotóxico parece ser um fator associado a maior risco de carcinogênese, embora as pesquisas sugiram maiores avaliações sobre o tema. Os trabalhos analisados nesta revisão sugerem associação dos agrotóxicos a impactos negativos para a saúde do homem e na incidência de doenças como diabetes mellitus ou neoplasias, entretanto reforçam a importância de mais estudos experimentais para consolidar tal hipótese. O fato de que a saúde dos trabalhadores rurais é afetada pela exposição aos agrotóxicos aponta a necessidade de medidas para redução de riscos na utilização dos produtos, visando a melhoria na qualidade do trabalho e evitando danos futuros a esses trabalhadores.

Este trabalho, portanto, espera estimular mais discussões sobre a exposição aos pesticidas e a incidência de outros tipos de neoplasias, propiciando visibilidade às questões relacionadas aos riscos eminentes da exposição inadequada a agrotóxicos através da criação de política públicas de segurança e proteção às populações potencialmente expostas a esses produtos. Apesar da relevante contribuição, alguns vieses foram observados ao final da pesquisa, tais como a classificação incorreta de exposição não diferencial, além do fato do câncer ser uma doença multifatorial. Em geral, a literatura é bem ilustrada no caso de tumores malignos de próstata, tireoide, linfoma e mama. Estudos que investiguem ainda mais a relação entre agrotóxicos e outras neoplasias são recomendados.

\section{REFERÊNCIAS}

ANVISA. AGENCIA NACIONAL DE VIGILÂNCIA SANITÁRIA. UFPR. Seminário MERCADO DE AGROTÓXICO E REGULAÇÃO, 2012. Brasília: Anvisa. Disponível em: https://bvsms.saude.gov.br/bvs/publicacoes/relatorio_nacional_vigilancia_populacoes_expost as_agrotoxicos.pdf. Acessado em: 12 de junho de 2021.

BA, Yue et al. Occupation and thyroid cancer: a population-based case-control study in Connecticut. Journal of occupational and environmental medicine/American College of Occupational and Environmental Medicine, v. 58, n. 3, p. 299, 2016.

BOCHNER, Rosany et al. Óbito ocupacional por exposição a agrotóxicos utilizado como evento sentinela: quando pouco significa muito. 2015. 
BORGES, Diego Echevenguá et al. O processo de institucionalização do uso de agrotóxicos entre pequenos cotonicultores brasileiros. 2018.

CARGNIN, Marcia Casaril dos Santos; ECHER, Isabel Cristina; SILVA, Djulia Rosa da. Fumicultura: uso de equipamento de proteção individual e intoxicação por agrotóxico. Revista de Pesquisa: Cuidado é Fundamental Online. Rio de Janeiro. vol. 9, n. 2 (abr./jun. 2017), p. 466-472, 2017.

CARNEIRO, Fernando Ferreira et al. Dossiê ABRASCO: um alerta sobre os impactos dos agrotóxicos na saúde. EPSJV/Expressão Popular, 2015.

CARNEIRO, Fernando Ferreira et al. Os impactos dos agrotóxicos no contexto do agronegócio. Embrapa Hortaliças-Capítulo em livro científico (ALICE), 2015.

CEZAR-VAZ, Marta Regina et al. Abordagem socioambiental na enfermagem: focalizando o trabalho rural e uso de agrotóxicos. Revista Brasileira de Enfermagem, v. 69, n. 6, p. 11791187, 2016.

CHA, Eun Shil; JEONG, Mihye; LEE, Won Jin. Agricultural pesticide usage and prioritization in South Korea. Journal of Agromedicine, v. 19, n. 3, p. 281-293, 2014.

DE MIRANDA, Natália Machado et al. Evidências dos efeitos dos agrotóxicos na carcinogênese. Revista da Graduação, v. 8, n. 1, 2015.

DE OLIVEIRA, Elton Carlos; DE ABREU, João Francisco; BARROSO, Leônida Conceição. A mortalidade por câncer na região metropolitana de belo horizonte: uma análise exploratória.

Revista Espaço e Geografia, v. 18, n. 3, 2015.

ENGEL, Lawrence S. et al. Pesticide use and breast cancer risk among farmers' wives in the agricultural health study. American Journal of Epidemiology, v. 161, n. 2, p. 121-135, 2017.

FREIRE, Carmen; KOIFMAN, Rosalina Jorge; KOIFMAN, Sergio. Serum levels of organochlorine pesticides in blood donors: A biomonitoring survey in the North of Brazil, 2010-2011. Science of the Total Environment, v. 598, p. 722-732, 2017.

GALVÃO, Taís Freire; PANSANI, Thais de Souza Andrade; HARRAD, David. Principais itens para relatar Revisões sistemáticas e Meta-análises: A recomendação PRISMA. Epidemiologia e Serviços de Saúde, v. 24, p. 335-342, 2015.

GANGEMI, Silvia et al. Occupational exposure to pesticides as a possible risk factor for the development of chronic diseases in humans. Molecular Medicine Reports, v. 14, n. 5, p. 44754488, 2016.

GONÇALVES, Glaciene Mary da Silva et al. Uso de agrotóxicos e a relação com a saúde na etnia Xukuru do Ororubá, Pernambuco, Brasil. Saúde e Sociedade, v. 21, p. 1001-1012, 2012.

GRABOIS, Marília Fornaciari et al. Completude da informação "Ocupação" nos Registros Hospitalares de Câncer do Brasil: bases para a vigilância do câncer relacionado ao trabalho.

Revista Brasileira de Cancerologia, v. 60, n. 3, p. 207-214, 2014. 
GRANGEIRO, Amanda Mesquita et al. Incidência e mortalidade por câncer de próstata no Tocantins e palmas, no período de 2010 a 2014. Revista de Patologia do Tocantins, v. 6, n. 1, p. 27-30, 2019.

GROSICKA-MACIĄG, Emilia et al. Modulation of antioxidant defense system by the dithiocarbamate fungicides Maneb and Zineb in Chinese hamster V79 cells and the role of Nacetyl-L-cysteine. Food and Chemical Toxicology, v. 60, p. 130-134, 2013.

INSTITUTO NACIONAL DE CÂNCER. Estimativa 2016: Incidência de Câncer no Brasil/ Instituto Nacional de Câncer José Alencar Gomes da Silva, Coordenação de Prevenção e Vigilância. Rio de Janeiro, 2016.

KAHL, Vivian Francília Silva; DA SILVA, Juliana; DA SILVA, Fernanda Rabaioli. Influence of exposure to pesticides on telomere length in tobacco farmers: A biology system approach.

Mutation Research/Fundamental and Molecular Mechanisms of Mutagenesis, v. 791, p. 19-26, 2016.

LEMARCHAND, Clémentine et al. Prostate cancer risk among French farmers in the AGRICAN cohort. Scandinavian Journal of work, environment \& health, p. 144-152, 2016.

LEÓN, Juan Manuel et al. Widespread occurrence and spatial distribution of glyphosate, atrazine, and neonicotinoids pesticides in the St. Lawrence and tributary rivers. Environmental Pollution, v. 250, p. 29-39, 2019.

LERRO, Catherine C. et al. A longitudinal study of atrazine and 2, 4-D exposure and oxidative stress markers among Iowa corn farmers. Environmental and molecular mutagenesis, v. 58, n. 1, p. 30-38, 2017.

LONDRES, Flavia. Agrotóxicos: um mal realmente necessário? MMA/Coleciona: fichário do Educador Ambiental, v. 1, n. 13 (maio 2015) Brasília, Edição Especial. 96 p., v. 1, n. 13,p. 23, 2015.

LOPES, Carla Vanessa Alves; ALBUQUERQUE, Guilherme Souza Cavalcanti de. Agrotóxicos e seus impactos na saúde humana e ambiental: uma revisão sistemática. Saúde em Debate, v. 42, p. 518-534, 2018.

MIRANDA, Celeni et al. A utilização de agrotóxicos nos municípios de Jataí e Perolândia (GO): embasamento legal e impacto na saúde pública. 2016.

MOHER, David et al. Preferred reporting items for systematic reviews and meta-analyses: the PRISMA statement. PLoS medicine, v. 6, n. 7, p. e1000097, 2009.

OLIVEIRA, Luã Kramer de et al. Processo sócio-sanitário-ambiental da poluição por agrotóxicos na bacia dos rios Juruena, Tapajós e Amazonas em Mato Grosso, Brasil 1. Saúde e Sociedade, v. 27, p. 573-587, 2018.

OMS. Organização Mundial da Saúde. Cancer. Disponível em: <http://www.who.int/mediacentre/factsheets/fs297/en/>. Acesso em: 25 de maio de 2019.

PLUTH, Thaís Bremm. Exposição à agrotóxicos e câncer. 2017. 
PLUTH, Thaís Bremm; ZANINI, Lucas Adalberto Geraldi; BATTISTI, Iara Denise Endruweit. Pesticide exposure and cancer: an integrative literature review. Saúde em Debate, Rio de Janeiro, v. 43, n. 122, p. 906-924, Setembro, 2019 . Disponível em <http://www.scielo.br/scielo.php?script=sci_arttext\&pid=S010311042019000300906\&lng=en\&nrm=iso>. Acesso em 22 de Março de 2021.

RIBEIRO, Daniel das Chagas de Azevedo et al. A resolução de problemas na Educação Básica: o processo de elaboração de problemas sobre a temática ambiental agrotóxicos. Encontro de Debates sobre o Ensino de Química (38.; 2018 out. 18-19, Canoas, RS). Anais. Canoas, RS: Universidade Luterana do Brasil (Ulbra), 2018 , 2018.

RIGOTTO, Raquel Maria; AGUIAR, Ada Cristina Pontes. Invisibilidade ou invisibilização dos efeitos crônicos dos agrotóxicos à saúde? Desafios à ciência e às políticas públicas.ipt. 2016.

SANTOS, Vilma Constancia Fioravante dos et al. O uso de agrotóxicos e suas implicações para a saúde humana e ambiental: enfrentamento local. Saúde coletiva, desenvolvimento e (in) sustentabilidades no rural. Porto Alegre: UFRGS, 2018. p. 181-95, 2018.

SILINS, Ilona; KORHONEN, Anna; STENIUS, Ulla. Evaluation of carcinogenic modes of action for pesticides in fruit on the Swedish market using a text-mining tool. Frontiers in pharmacology, v. 5, p. 145, 2014.

SILVA, Eveline Fronza da et al. Prevalência de morbidades e sintomas em idosos: um estudo comparativo entre zonas rural e urbana. Ciência \& Saúde Coletiva, v. 18, n. 4, p. 1029-1040, 2013.

SILVA, João Francisco Santos da et al. Correlação entre produção agrícola, variáveis clínicasdemográficas e câncer de próstata: um estudo ecológico. Ciência \& Saúde Coletiva, Rio de Janeiro, v. 20, n. 9, p. 2805-2812, setembro, 2015. Disponível em

<http://www.scielo.br/scielo.php?script=sci_arttext\&pid=S1413$81232015000902805 \& \operatorname{lng}=$ en\&nrm=iso>. Acesso em 22 de setembro de 2020.

SMITH, Adam M. et al. 2, 4-Dichlorophenoxyacetic acid (2,4-D) and risk of non-Hodgkin lymphoma: a meta-analysis accounting for exposure levels. Annals of Epidemiology, v. 27, n. 4, p. 281-289. e4, 2017.

TEYSSEIRE, Raphaëlle et al. Assessment of residential exposures to agricultural pesticides: A scoping review. PloS one, v. 15, n. 4, p. e0232258, 2020. 


\section{Declaração de Interesse}

Os autores declaram não haver nenhum conflito de interesse

\section{Financiamento}

Financiamento próprio

\section{Colaboração entre autores}

$O$ presente artigo foi escrito pelo J. B. F. sob orientação dos professores L. F.G. e P. A. F. S., projetado e concluído no Mestrado Profissional em Ensino de Ciências da Saúde e do Ambiente (PROCISA) da Faculdade Dinâmica do Vale do Piranga (FADIP).

Ambos os autores cuidaram da parte dissertativa do artigo. 